\title{
AFUERA: LA DESAPARICIÓN DEL UNDERGROUND COMO SÍNTOMA
}

\author{
Enrique Hurtado Mendieta \\ Universidad del País Vasco / Euskal Herriko Unibertsitatea. Dpto Escultura y Arte y Tecnología
}

\section{Resumen}

Este artículo analiza la situación de parálisis que desde finales de los años 90 parece sufrir la cultura popular, especialmente la música. Para ello examinamos el papel de las transformaciones estilísticas que ésta sufrió a partir de los años 50 y 60 y el contexto sociocultural en el que suceden. Posteriormente analizamos la manera en que la digitalización y las redes han cambiado las dinámicas en las que se sustentaban estas transformaciones y sus implicaciones, a fin de plantear una crítica al análisis que diferentes autores realizan sobre esta falta de vitalidad en la cultura popular actual.

\section{Palabras clave: MODERNISMO POPULAR; MÚSICA; UNDERGROUND}

\section{OUTSIDE: THE DISAPPEARANCE OF THE UNDERGROUND AS A SYMPTOM}

Abstract

This article analyses the situation of paralysis that popular culture, especially music, seems to have been suffering since the end of the 1990s. To do so, we examine the role of the stylistic transformations it underwent from the 1950s and 1960s onwards and the socio-cultural context in which they occurred. We then analyse the way in which digitalisation and networks have changed the dynamics underpinning these transformations and their implications, in order to critique the analysis that different authors make of this lack of vitality in today's popular culture.

Keywords: POPULAR MODERNISM; MUSIC; UNDERGROUND

Hurtado Mendieta, Enrique. 2021. "Afuera: La desaparición del underground como síntoma". AusArt 9 (1): 117-126. D0I: 10.1387/ausart.22651

\section{AUSART}




\section{INTRODUCCIÓN}

Nos volvimos outsiders, y comentábamos todo mirando desde afuera. Es algo que a mucha gente le cuesta hacer hoy. Porque no existe un "afuera".

(Genesis P-Orridge en Stubbs 2019, 376)

El concepto de underground surge durante los años 60 ligado a la contra-cultura, la insurgencia política y a la cultura popular. El underground es un ámbito sociocultural aparte donde ocurren prácticas alternativas y diferentes a las hegemónicas, convencionales o mainstream y habitualmente ha sido considerado como un espacio al margen de la industria cultural y del mercado. Si bien durante décadas el underground funcionó como una especie de laboratorio donde se desarrollaron múltiples tendencias culturales que alcanzaban amplia visibilidad, en los últimos años parece haber desaparecido, tal como plantean diversos autores y como veremos más adelante. La actual inexistencia de este afuera sería un síntoma del agotamiento de las dinámicas socioculturales que se iniciaron a mitad del siglo XX y denota que nos encontramos en un cambio de ciclo.

Después de la Segunda Guerra Mundial se produce una diseminación de la cultura popular anglosajona por casi todo el globo gracias al despliegue de las tecnologías de transmisión de información, como la televisión, la radio o el satélite, y el perfeccionamiento de las tecnologías de grabación y reproducción de sonido. Esto, junto con otros factores políticos y socio-económicos, como la aparición del estado de bienestar en Europa y la bonanza económica tras la posguerra, propició que se desarrollara una cultura popular global que tuvo a la música como uno de sus principales vehículos. Anteriormente ya habían comenzado a perfilarse procesos similares alrededor del jazz, pero es con el pop y el rock cuando finalmente esta cultura de base popular, por primera vez, se convierte en un fenómeno global extendiéndose por la mayor parte del mundo.

Dentro de este contexto los movimientos y tendencias se sucedieron durante varias décadas en diversos ámbitos de la cultura (música, moda, diseño, etc.) mediante continuos ciclos de ruptura y reconstrucción que se alternaron con gran rapidez a la vez que se desarrollaban y popularizaban nuevas y continuas transformaciones estéticas. Esta dinámica fue especialmente relevante en la música popular donde infinidad de géneros y subgéneros se fueron superando unos a otros en un proceso acelerado de cambio continuo. El gran dinamismo 
creativo que tuvo la cultura en estas décadas estuvo vinculada a movimientos socio-políticos que reivindicaron a la cultura popular global como un vehículo adecuado para el desarrollo de modernidad a la vez que como motor de cambio social. Esto dio lugar a que por un momento quedara atrás la concepción de la cultura de vanguardia como propiedad exclusiva y a la vez como sello distintivo de la élite social. Esta puesta en valor de la cultura popular como germen y catalizador de cambios sociales profundos está vinculada a lo que Fisher ha venido en llamar 'modernismo popular' $(2018,50)$, un proceso en el que las ideas de vanguardia y la cultura popular interactuaron buscando la transformación de la sociedad. Sin embargo "los futuros que el modernismo popular nos preparó para esperar [...] nunca se materializaron" (ibíd., 55). Estos movimientos sociales tuvieron su punto álgido a finales de los años 60 y principios de los 70 para terminar cerrándose en falso y provocar posteriormente una larga resaca desde finales de los años 70 y a través de los años 80 . La ansiada transformación social derivó en una sucesión de cambios que, aunque afectaron a diversos ámbitos de la sociedad, no llegaron a amenazar a la esencia del sistema, sino que en gran medida acabaron siendo aprovechados por éste. Finalmente, a partir de finales de los años noventa, según algunos autores, esta sucesión de mutaciones se ralentiza y la cultura popular cae en un estado de ensimismamiento en el que parece que no se produce ya ningún avance (Mark Fisher 2014; Reynolds 2012, 417; Stubbs 2019, 50; Berardi 2014, 127; Reynolds 2013, 202). Algo impide que se generen nuevas mutaciones y cambios y este proceso que anteriormente fue tan fructífero, especialmente en el ámbito de la música, ahora parece haberse detenido. Un vistazo el cartel del festival Primavera Sound de 2022 es suficiente para ilustrar esta idea. La mayoría de los cabezas de cartel son bandas o solistas con décadas de carrera a sus espaldas (Beck, Massive Attack, Gorillaz, Nick Cave, Pavement) o con fortísimas resonancias a estilos del pasado (The Strokes, Tame Impala, Interpol). Esto no quiere decir que no haya novedades, sino que estas son casi inapreciables si las comparamos con las que ocurrieron en la música popular el periodo 1965-1985, por ejemplo.

\section{DESARROLLO}

Este incesante movimiento de ruptura y reconstrucción que caracterizó a la música popular global durante décadas, y que para algunos define la esencia misma del sistema capitalista, se basaba en la existencia de un afuera, 
una periferia, un contexto social diferenciado, una comunidad 'fuera del radar' donde germinaban nuevas ideas y conceptos de forma autónoma, impredecible y fuera de control, pero siempre por debajo de cierto umbral y sin llegar a plantear una amenaza o alternativa al sistema mismo. Esto es especialmente claro a partir de los años 70 con la llegada del punk. Los nuevos paradigmas se desarrollaban libremente y hasta madurar lo suficiente para que llegado el momento fueran explotados, absorbidos y reutilizados por la maquinaria de la industria musical y cultural, siempre después de haber sido neutralizados y despojados de los aspectos excesivamente transformadores que pudieran contener. Algunos de los conceptos desarrollados en este entorno alternativo llegaban incluso a convertirse en hegemónicos dentro de la cultura mainstream una vez habían sido controlados y asimilados. Estas mutaciones se repetían una y otra vez en un proceso de destrucción/construcción donde se rompía con los esquemas existentes pero donde a la vez se reutilizaba elementos de la situación anterior en la construcción de lo nuevo.

Si miramos a los dos países por excelencia desde los que la cultura popular global se diseminó, EEUU y el Reino Unido, los afueras son lugares marcados principalmente por la diferencia social de clase. En el caso de la música pop rock estadounidense uno de esos 'afueras' fue la cultura afroamericana y por otro lado la cultura de los white trash, las clases bajas de ascendencia europea. En el caso del Reino Unido, estaría la cultura de la clase trabajadora como un espacio diferenciado así como la cultura de los west-indians, descendientes de los inmigrantes de las colonias británicas en el Caribe $\square$ que llegaron a la metrópolis tras la Segunda Guerra Mundial. En estos espacios al margen se generan incontables mutaciones y tendencias musicales que alimentan posteriormente a la industria. Quizás uno de los últimos movimientos que parecen seguir este patrón de asimilación de lo alternativo, o quizás uno de los más paradigmáticos, sea el grunge. A finales de los años ochenta, la escena del rock alternativo de la ciudad de Seattle, donde el grunge floreció, se constituyó como un afuera donde se produjo un caldo de cultivo ideal para que un contexto cultural diferente pudiera nacer y desarrollarse lejos de la atención de la prensa y la industria musical. Una vez estuvo maduro, se popularizó y fue finalmente asimilado y aprovechado por la maquinaria cultural en diversos ámbitos como la moda, el cómic o principalmente la música. Con un marcado carácter de white trash en sus inicios, el grunge partía de una reutilización de elementos del rock anterior, reivindicando artistas de los años 70 muy diversos como Black Sabbath o Neil Young, a la vez que planteaba una estética nueva donde estas referencias se re-elaboraron a partir de la experiencia del punk y el hardcore. El grunge tenía sus raíces en el circuito del rock alternativo estadounidense que había nacido con el punk y que durante años 
fue madurando y creciendo lentamente de la mano de las radios universitarias y de sellos musicales independientes como SST, que publicó trabajos de grupos decisivos para la existencia del grunge como Black Flag, Soundgarden, Sonic Youth o Dinosaur Jr. Es a esta genealogía a la que atiende en su título 1991: The year that punk broke, el documental sobre la gira europea de Sonic Youth durante el verano de 1991 (Markey, 1992). En él, se pueden ver actuaciones de Sonic Youth junto con Nirvana o Dinosaur Jr, así como diversas situaciones del día a día de esa gira.

No obstante, actualmente nuestras circunstancias en lo relativo a las formas de generación, maduración y difusión de movimientos y tendencias culturales son diferentes a décadas de los sesenta a los noventa. Al analizar la situación actual de parálisis en la música popular, Reynolds $(2012,91)$ plantea que uno de los factores que dan lugar a esta inmovilidad es el acceso cada vez más instantáneo y exhaustivo a la información del pasado que hoy día tenemos gracias a las tecnologías digitales de almacenamiento, acceso y transmisión de datos. Mediante estas tecnologías el pasado se encuentra disponible en su totalidad (o eso parece al menos) para ser explorado en todo momento y todo lugar. Sin embargo toda la información está disponible al mismo nivel, sin ningún tipo de contexto ni diferenciación, y de manera simultanea; Duke Ellington, Napalm Death y Caterina Barbieri a la vez, pura información sin cuerpo. En relación a este fenómeno de superabundancia y saturación de información, Franco "Bifo" Berardi plantea que actualmente la cantidad de datos que se transmiten y su velocidad de transmisión es tan alta que está más allá de la capacidad humana de asimilación $(2014,146)$. Esto da lugar a que sea imposible comprender el presente porque no podemos usar ni la voluntad ni la razón para hacerlo (Berardi 2017, 36). "El entorno, acelerado por el poder de la tecnología, hoy en día excede cualquier posibilidad de medida humana" (ibíd., 44). Aparte de estas causas que plantean tanto Berardi como Reynolds podemos intentar rastrear otras.

En este sentido es interesante mirar a la música y la cultura a la luz de esta afirmación que hace Lovink respecto al Ilamado capitalismo de vigilancia: "Esta es la razón por la cual los terroristas ya no pueden crear células y reunirse, la máquina de predicción algorítmica los detectará inmediatamente" $(2019,244)$. Todos los aspectos de nuestra sociedad resultan afectados hoy día por esta vigilancia algorítmica global, y la música y la cultura en general no son una excepción. Esta maquinaria algorítmica descubre y absorbe actualmente las nuevas tendencias y movimientos antes de que tengan tiempo para organizarse, desarrollarse y madurar con lo que el binomio ruptura/renovación que 
empujó los cambios durante décadas, se encuentra cortocircuitado porque el underground se ha desvanecido. Esto provoca que la acción colectiva en la creación de un contexto o escena local resulte casi inútil, más allá de espacios radicalmente alternativos e invisibles, y que la única estrategia viable parezca ser la del individualismo. Quizás esto explique por qué en los últimos años están surgiendo en la música popular más solistas que nunca mientras el formato de grupo parece haberse quedado en un segundo plano o directamente obsoleto. Este fenómeno también está relacionado con los cambios en las tecnologías de producción musical que han hecho redundantes a los músicos del grupo tradicional. Cajas de ritmo, samplers, auto-tune, secuenciadores, editores de audio, plugins, el reciente y cada vez más efectivo uso de sistemas de inteligencia artificial en los procesos de composición, generación de arreglos y masterización, nos llevan a un escenario 'Ikea' en el que el conocimiento, la maestría técnica, pero sobre todo la colaboración con otros músicos, es cada vez menos necesaria y más evitable. Por otro lado, aunque siempre ha existido, cada vez es más crucial el trabajo del productor-compositor de manera que el cantante-solista pasa a ser un mero actor que interpreta las creaciones de otros. Este fenómeno da lugar a una progresiva homogeneización de la música existente a todos los niveles (compositivo, tímbrico, estilístico, interpretativo, etc.) en el que la música popular cada vez adopta más paradigmas de la industria del cine: star-system, montaje tipo collage, procesos industriales de trabajo, etc. La evolución artística de estos solistas sigue frecuentemente un patrón similar: un inicio prometedor y sugerente da paso a una rapidísima asimilación por parte de la industria que redirige su carrera hacia formas y estilos mucho más normales e inofensivos pero con un público potencial mucho más amplio. Junto con esta asimilación se produce en muchos casos una integración casi total con la industria pop de creación de estrellas y con la publicidad y la moda. En algunos casos esta transición da resultados más interesantes que en otros, ya que a veces la originalidad y calidad artística se queda por el camino. Por citar unos ejemplos de este tipo de transformación exprés: Kilo Kish, Grimes o Tommy Genesis. En contraste con el momento actual, en las décadas anteriores a los años 2000 existía una separación mucho más clara entre lo alternativo y lo hegemónico y generalmente los artistas operaban en uno $u$ otro de estos dos mundos. En contadas ocasiones algunos daban el salto entre ellos, un fenómeno relativamente puntual que se daba normalmente en esos momentos que hemos descrito anteriormente, en los que una tendencia pasaba a ser explotada por la industria. Sin embargo, hoy día casi no existe diferencia alguna entre ambos mundos porque el espacio alternativo prácticamente ha desaparecido y la transición entre ellos es instantánea o directamente inexistente, como ha ocurrido con Billie Eilish. En ocasiones se da el caso donde incluso, a la manera del gato de Schroedinger, un artista 
puede paradójicamente estar a la vez underground y mainstream (https://www. youtube.com/watch?v=3J0Ej4Nk-K8).

Por otro lado, y como crítica a los razonamientos de algunos de estos autores que mencionábamos antes y que describen la actual situación que ellos califican de bloqueo, pero también como una forma de contextualizar y entender mejor su análisis, es importante señalar que los ejemplos utilizados por algunos de ellos para ilustrar sus planteamientos están habitualmente localizados en una época y lugar concretos (principalmente el Reino Unido entre finales de los años setenta y los años ochenta). Esto es especialmente llamativo en el caso de Reynolds y Fisher, pero también en Stubbs. Con todo, este último ya aborda esta problemática cuando dice que "El único problema con la escuela de hauntología de Ghost Box es que esos 'recuerdos' que deliberadamente implanta solo son resonantes para algunos de nosotros: los nacidos en cierto momento (entre 1955 y 1970, digamos) y cierto lugar (Reino Unido)" (Stubbs 2019 , 398). A continuación, sin embargo, obvia casi totalmente la polifacética y efervescente escena de la música electrónica y experimental basada en ordenadores que se desarrolló desde finales de los años 90 en todo el mundo una vez que los ordenadores fueron asequibles y el software maduró mientras incorporaba funcionalidades desarrolladas en la investigación académica relacionada con la música contemporánea (por ejemplo. http://www. pure-data.info). Estas escenas musicales, que han funcionado a nivel global, han sido interesantes especialmente por la hibridación de las músicas de tradición 'culta', como la contemporánea y experimental, y la música popular y han dado lugar a múltiples y diversas escenas, géneros y estilos en una manera similar a la efervescencia del pop y el rock durante sus años dorados pero con una visibilidad y popularidad mucho menor.

Deberíamos plantearnos entonces que, si bien las ideas y análisis de estos autores son interesantes para entender la situación en la que estamos, tienen un sesgo generacional y cultural. En un intento de superar su análisis, tenemos que considerar que los canales y tecnologías de transmisión de la información actuales no son ya los mismos que existían en la época en la que se desarrollaron los movimientos musicales que estos autores usan como base de su análisis. La hegemonía de la prensa musical especializada y las televisiones y radios estatales, que permitió y facilitó la difusión de la cultura pop hasta los años noventa, se rompió totalmente con la llegada de Internet. Esta prensa especializada, que se desarrolla principalmente durante los años 80 tras la explosión del punk, era en parte usada por la industria, por un lado como una forma de promocionar sus productos, y por otro, como un sistema para descu- 
brir nuevas tendencias y artistas que llevar al mercado. La llegada de internet, el mp3 y la tecnología P2P de distribución de archivos provocaron una fragmentación y un increíble aumento de la rapidez con la que circula la información (Reynolds 2013, 206, 207). Este es un proceso que no ha parado de crecer desde la popularización de Internet hasta la actualidad. A su vez, y como ya apuntábamos antes, el volumen de datos disponibles es cada vez mayor. Estos factores se relacionan con los planteamientos de Berardi respecto a la velocidad y forma de transmisión de información que mencionábamos antes. En estas circunstancias de aceleración y multiplicidad el análisis es imposible y el pesimismo de Reynolds y Fisher refleja en gran medida esta pérdida de la capacidad de entender el presente y como consecuencia la capacidad de tener un efecto transformador sobre él. Sin embargo, quizás ahora mismo el problema no sea tanto que no se generen cambios y novedades en la cultura, sino que estas nuevas ideas permanecen invisibles e inaccesibles, unas porque son absorbidas y neutralizadas demasiado rápido por la industria cultural, otras porque no llegan a darse a conocer al ser absolutamente subterráneas, efímeras e inestables por lo que resultan invisibles a una mirada 'tradicional' basada en los parámetros de análisis que se usaban hasta los años noventa y que ya no son válidos. Ante una situación inabarcable como la actual es normal que se den repliegues hacia épocas más fáciles de entender porque la música "Es también un medio extremadamente eficaz de exploración del pasado, en un momento en que el presente no responde ya a las necesidades de todos" (Attali [1977] 1995, 149). La tentación retro siempre estuvo en el ADN de la música pop, como bien analiza Reynolds (2012), solo que en los últimos años ha adquirido un gran peso y en gran medida nos ciega.

A pesar de lo planteado por estos autores, y del pesimismo que rodea su análisis, podemos encontrar en internet una legión de músicos realizando trabajos altamente innovadores en muchos aspectos, que fieles a la tradición de la cultura popular, recogen las influencias del pasado pero realizan una apropiación y actualización de estas referencias. Proyectos como Klein, Coby Sey, Micachu and the Shapes, Moor Mother, Maria W. Horn y muchísimos otros mantienen la innovación y el cambio en marcha. Solo que ahora son mucho más difíciles de encontrar que nunca. 


\section{CONCLUSIÓN}

Hemos visto como a partir de los años 50 se inaugura una época de transformaciones sociales de la mano de la aparición en la cultura de un proceso que algunos denominan 'modernismo popular'. Esto ocurre en un contexto de globalización de la cultura que en música popular se caracteriza por una gran efervescencia estilística basada en la existencia de un afuera underground donde los cambios germinan. A partir de finales de los años 60 esta época de transformaciones sociales se va cerrando en falso sin llegar a producir un cambio social profundo y desde finales de los 70 se inaugura una larga resaca, cuando finalmente el binomio underground/mainstream se pone definitivamente al servicio de la industria cultural y musical. A final de los años 90 se produce un agotamiento como consecuencia de la aceleración y fragmentación que provocan, entre otras causas, la digitalización y las redes. Esta situación impide la existencia de un afuera lo que paraliza el proceso de cambio estilístico en la música. Al analizar esta parálisis vemos que la forma en que la cultura y los cambios que en ella se desarrollan y diseminan han ido cambiado radicalmente desde la popularización de Internet lo que nos ha llevado de una situación de relativa escasez de información a otra de superabundancia de ella. En un momento en el que todo está disponible instantáneamente desde cualquier lugar, la existencia de un afuera ya no es posible en la manera en lo fue anteriormente. Ante esta situación comprobamos que es inútil analizar la realidad con los parámetros del pasado ya que nos lleva a aplicar sesgos culturales o generacionales en nuestro análisis. Necesitamos desarrollar nuevas herramientas para entender una realidad cada vez más caótica y fragmentada y poder plantear estrategias que permitan que nuestra práctica tenga un efecto real y transformador sobre nuestro presente y nuestro futuro y que no solo se quede en un mero cambio formal. Esto no va a ocurrir defendiendo un retorno a un pasado dorado o renegando del uso de las tecnologías actuales, sino buscando nuevas formas de construcción de espacios y contextos alternativos que se adapten a las circunstancias actuales y consigan escapar al capitalismo de vigilancia. Para esta labor deberíamos de tener en cuenta a Berardi cuando nos dice que "[...] la relevancia o efectividad de la acción humana ya no sucede en el nivel del conocimiento racional, de la decisión política y la voluntad, sino en el nivel de la intuición, la imaginación y la sensibilidad" $(2014,127)$. 


\section{Referencias bibliográficas}

Attali, Jacques. (1977) 1995. Ruidos: Ensayo sobre la economía política de la música. Traducción de José Martín Arancibia. Ciudad de México: Siglo XXI

Berardi, Franco (Bifo). 2014. Después del futuro: Desde el futurismo al cyberpunk; El agotamiento de la modernidad. Traducción y prólogo de Giuseppe Maio. Madrid: Enclave de Libros

Berardi, Franco (Bifo). 2017. Fenomenología del fin: Sensibilidad y mutación conectiva. Traducido por Alejanda López Gabrielidis. Buenos Aires: Caja Negra

Fisher, Mark 2014. "The slow cancellation of the future". Vídeo de Youtube, 46:14, 22 mayo. https://www.youtube.com/watch?v=aCgkLICTskQ

Fisher, Mark. 2018. Los fantasmas de mi vida: Escritos sobre depresión, hauntología y futuros perdidos. Traducido por Pablo Schanton. Buenos Aires: Caja Negra

Lovink, Geert. 2019. Tristes por diseño: Las redes sociales como ideología. Traducido por Matheus Calderón Torres. Bilbao: Consonni

Reynolds, Simon. 2012. Retromanía: La adicción del pop a su propio pasado. Traducido por Teresa Arijón \& Pablo Schanton. Buenos Aires: Caja Negra

Stubbs, David. 2019. Sonidos de Marte: Una historia de la música electrónica. Traducido por Tadeo Lima. Buenos Aires: Caja Negra 